\title{
CARACTERÍSTICAS DE PRÁTICAS DE EDUCAÇÃO EM SAÚDE REALIZADAS POR ESTUDANTES DE ENFERMAGEM
}

\author{
Francisco Gilberto Fernandes Pereira ${ }^{1}$, Sâmia Jucá Pinheiro², Joselany Áfio Caetano³, Márcia Barroso Camilo \\ de Ataíde
}

\begin{abstract}
RESUMO: Objetivou-se descrever características de práticas de Educação em Saúde realizadas por estudantes de graduação em enfermagem.Trata-se de estudo descritivo exploratório realizado entre fevereiro e junho de 2015, em uma instituição privada de ensino superior em Fortaleza, estado do Ceará, que analisou, por meio de um formulário padronizado, 60 atividades desenvolvidas por estudantes do nono e décimo período do curso. As principais técnicas educativas utilizadas foram: palestra, jogos educativos e associação de técnicas diversas; realizadas principalmente na atenção primária 38 (63\%). A temática geral variou de acordo com o nível de atenção em saúde, porém a orientação de cuidados/tratamentos a grupos específicos foi mais comum em 25 (42\%) das ações educativas. Conclui-se que as práticas de Educação em Saúde seguem um modelo eminentemente preventivo, marcadas pela presença do lúdico.
\end{abstract}

DESCRITORES: Educação em saúde; Estudantes de enfermagem; Educação em enfermagem.

\section{CHARACTERISTICS OF NURSING STUDENTS' HEALTH EDUCATION PRACTICES}

\begin{abstract}
The objective was to describe characteristics of undergraduate nursing students' Health Education practices. A descriptive and exploratory study was undertaken between February and June 2015 at a private higher education institution in Fortaleza, state of Ceará, Brazil. Using a standardized form, 60 activities students developed in the ninth and tenth course terms were analyzed. The main educational techniques used were: lecture, educative games and association of several techniques; mainly performed in primary care $38(63 \%)$. The general theme varied according to the health care level, but the care/treatment orientation to specific groups was more common in $25(42 \%)$ of the educative actions. In conclusion, the Health Education practices follow an eminently preventive model, marked by the presence of the playful.
\end{abstract}

DESCRIPTORS: Health education; Nursing students; Nursing education.

\section{CARACTERÍSTICAS DE PRÁCTICAS DE EDUCACIÓN EN SALUD DESARROLLADAS POR ESTUDIANTES DE ENFERMERÍA}

RESUMEN: La finalidad fue describir características de prácticas de Educación en Salud desarrolladas por estudiantes de pregrado en enfermería. Fue desarrollado un estudio descriptivo exploratorio entre febrero y junio de 2015, en una institución privada de educación superior en Fortaleza, estado de Ceará, Brasil. Mediante un formulario estandarizado, fueron analizadas 60 actividades desarrolladas por estudiantes del nono y décimo período del curso. Las principales técnicas educativas utilizadas fueron: conferencia juegos educativos y asociación de técnicas diversas; desarrolladas principalmente en la atención primaria 38 (63\%). La temática general varió según el nivel de atención en salud, pero la orientación de cuidados/tratamientos a grupos específicos fue más común en $25(42 \%)$ de las acciones educativas. Se concluye que las prácticas de Educación en Salud siguen un modelo eminentemente preventivo, marcadas por la presencia del lúdico.

DESCRIPTORES: Educación en salud; Estudiantes de enfermería; Educación en enfermería.

${ }^{1}$ Enfermeiro. Mestre em Enfermagem. Docente de Enfermagem da Universidade Federal do Piauí. Picos, PI, Brasil.

Enfermeira. Mestre em Cuidados Clínicos. Docente de Enfermagem do Centro Universitário Estácio do Ceará. Fortaleza, CE, Brasil.

${ }^{3}$ Enfermeira. Doutora em Enfermagem. Docente do Programa de Pós-graduação em Enfermagem da Universidade Federal do Ceará. Fortaleza, CE, Brasil.

${ }^{4}$ Enfermeira. Doutora em Enfermagem. Docente da Universidade de Fortaleza. Fortaleza, CE, Brasil

Autor Correspondente:

Francisco Gilberto Fernandes Pereira

Universidade Federal do Piauí

R. Professor Vicente Silveira, 100 - 60410-322 - Fortaleza, CE, Brasil

E-mail: gilberto.fp@hotmail.com
Recebido: 13/12/2015

Finalizado: $15 / 03 / 2016$ 


\section{INTRODUÇÃO}

Por muito tempo o cuidado à saúde teve seu referencial no modelo clínico, com a atenção prestada às pessoas em uma dimensão biológica e individual. Porém, a partir da década de 70, surgiu uma nova concepção de saúde, que não se limitava apenas em um enfoque voltado para a cura da doença, mas direcionava a atenção para prevenção de doenças e promoção da saúde ${ }^{(1)}$.

Entre as estratégias que visam fortalecer a promoção da saúde está a educação em saúde, que foca à conscientização, sensibilização e mobilização individual ou coletiva para o enfrentamento de situações que interferem na qualidade de vida das pessoas, sejam relativos a fatores individuais (intrínsecos) ou ambientais (extrínsecos) ${ }^{(2)}$.

Por sua magnitude, a educação em saúde deve ser entendida como importante vertente à prevenção, e que na prática deve estar preocupada com a melhoria das condições de vida e de saúde das populações ${ }^{(3)}$.

Para alcançar níveis adequados de saúde, as pessoas precisam saber identificar e satisfazer suas necessidades básicas, e serem subsidiadas por políticas públicas que permitam o alcance de melhor bem-estar. Também devem ser capazes de adotar mudanças de comportamentos, práticas e atitudes, além de dispor dos meios necessários à operacionalização dessas mudanças. Neste sentido, a educação em saúde significa a contribuição na aquisição de autonomia para identificar e utilizar as formas e os meios para preservar e melhorar a vida ${ }^{(3)}$.

Nesta concepção teórica, a equipe de saúde deve atuar na perspectiva de empoderar o usuário, e assim favorecer sua participação de forma ativa e consciente no cuidado à saúde. O conhecimento adquirido, associado ao conjunto dos determinantes sociais em saúde, permitem que os indivíduos tenham condições de fazer escolhas que promovam a saúde ou, ao contrário, predisponham à doença ${ }^{(4)}$.

Considerando que a educação em saúde está relacionada à aprendizagem, desenhada para alcançar a saúde, torna-se necessário que seja voltada a atender à população de acordo com sua realidade, uma vez que deve provocar conflito nos indivíduos, criando oportunidade da pessoa pensar e repensar a sua cultura, e ela própria transformar a sua realidade ${ }^{(5)}$.

Embora esteja havendo intensa incorporação tecnológica no campo da saúde nas últimas décadas, é preciso sensibilizar os estudantes da área de saúde, particularmente na área de enfermagem, de que a profissão tem a educação como um de seus eixos de trabalho. Essa sensibilização deve fomentar o cuidado de forma mais compartilhada e negociável, contemplando as necessidades dos sujeitos, sejam individuais ou coletivas ${ }^{(6)}$.

A presença da universidade para a sociedade tem papel importantíssimo, pois se acredita que os alunos que frequentam a academia fortalecem o desenvolvimento de seus conhecimentos e favorecem a realização de práticas educativas durante o ensino, fazendo com que os aprendizes pratiquem troca de conhecimento com a comunidade e, sendo assim, ajude a esclarecer suas dúvidas e buscar soluções conjuntas para os problemas apresentados conforme a realidade encontrada ${ }^{(7)}$.

A educação não está vinculada somente à atenção primária, e sim a todos os níveis de atenção à saúde. Seja na comunidade ou no hospital, a educação está sempre presente como ferramenta indispensável para o trabalho do enfermeiro. Perceber o caminho da profissão nessa nova perspectiva de enfermagem não só curativa, como também preventiva, é essencial na formação do acadêmico ${ }^{(8)}$.

Portanto, objetivou-se descrever as características das práticas de educação em saúde realizadas por estudantes de enfermagem.

\section{- MÉTODO}

Estudo descritivo, exploratório com abordagem quantitativa, realizado entre os meses de fevereiro e junho de 2015, em instituição de ensino superior privada, em Fortaleza, estado do Ceará. Nesta instituição, o bacharelado em enfermagem tem duração de cinco anos, com uma grade curricular 
composta de carga horária teórica e prática em laboratórios de simulação realística e na rede assistencial do município de Fortaleza (Unidades Básicas de Saúde, clínicas e hospitais), a partir do quarto período.

A execução das atividades educativas no ambiente externo à instituição de ensino precede um planejamento que é realizado geralmente por grupos de seis estudantes, que com o auxílio do docente de disciplina correspondente selecionam o grupo-alvo da intervenção educativa, seu local de realização, o tempo de execução, a definição de papéis e responsabilidades dos membros da equipe, e as possíveis técnicas que serão utilizadas.

Para operacionalização da coleta de dados, foi solicitado aos estudantes que apresentassem o planejamento, por escrito, da atividade educativa aos seus respectivos professores, que o encaminharam via eletrônica para o pesquisador. No total foram recebidas 60 atividades, sendo 38 do nono período e 22 do décimo, as quais compuseram a amostra deste estudo, pois satisfizeram aos critérios de inclusão: ser planejada e executada por estudantes do nono ou décimo período; e, conter as informações completas para o preenchimento do instrumento de coleta. A partir daí os dados foram coletados por meio da aplicação de um formulário que permitiu caracterizar o cenário da atividade educativa, os tipos de técnicas utilizadas e classificação da temática geral que foi abordada junto à clientela.

Convém ressaltar que todas as práticas educativas planejadas foram executadas pelos respectivos idealizadores com a supervisão de docentes da instituição de ensino.Todas as informações obtidas destas práticas foram organizadas em um banco de dados, no programa Statistical Software for the Social Science19.0 (SPSS) para julgamento exploratório e estatístico, por meio de distribuição descritiva dos dados.

Foram respeitados todos os aspectos bioéticos de acordo com a Resolução 466/12 ${ }^{(9)}$ do Ministério da Saúde, mediante autorização n. 889.504, emitido pelo Comitê de Ética em Pesquisa com Seres Humanos do Centro Universitário Estácio do Ceará.

\section{RESULTADOS}

A realização de atividades de educação em saúde esteve presente tanto nas disciplinas de Estágio Supervisionado I, II e III, que compreendem as áreas amplas de nível primário e os programas específicos da atenção básica no âmbito do Sistema Único de Saúde (SUS), como também no Estágio Supervisionado IV, V e VI, que por sua vez abrangem os níveis secundário e terciário, e portanto com maior inserção em serviços de saúde para atendimento de situações específicas e hospitais.

Assim, foi verificado que devido à multiplicidade e heterogeneidade dos públicos-alvo, bem como as condições do espaço físico onde essas atividades foram desenvolvidas, os estudantes utilizaram diversas técnicas para facilitar as ações educativas junto à clientela, com destaque para palestras e jogos educativos com $12(20 \%)$ cada. Identificou-se que em algumas situações houve associação de técnicas $(12-20 \%)$ principalmente quando a intervenção tinha como foco o público infantil. Esses dados estão expressos na Tabela 1.

Com relação ao nível de atenção em saúde onde as atividades educativas foram realizadas, identificou-se que a atenção primária foi comum em 38 (63\%), conforme demonstrado na Tabela 2. A maior ocorrência neste nível de atenção pode ser explicada pelo fato de que as disciplinas do nono período, que são realizadas em serviços de rede básica, possuem alinhamento metodológico de trabalho com as premissas do sistema de saúde brasileiro, que estimula sobremaneira atividades de educação em saúde nesta esfera de serviço.

Constatou-se que a orientação de cuidados/ tratamentos a grupos específicos foi a temática geral mais abordada pelos estudantes 25 (42\%),
Tabela 1 - Tipos de técnicas utilizadas para realização das atividades de educação em saúde por estudantes de enfermagem. Fortaleza, CE, Brasil, 2015

\begin{tabular}{lc} 
Técnica utilizada & $\mathbf{n}(\%)$ \\
\hline Palestra & $12(20)$ \\
\hline Dinâmica de grupo & $10(16)$ \\
\hline Jogos educativos & $12(20)$ \\
\hline Práticas simuladas & $6(10)$ \\
\hline Teatro & $8(14)$ \\
\hline Associação de técnicas & $12(20)$ \\
\hline Total & $60(100)$
\end{tabular}


ao passo que a discussão sobre comportamentos saudáveis apareceu com 14 (23\%), segundo dados apresentados na Tabela 2. Os grupos específicos aqui referidos foram basicamente aqueles que possuem programas governamentais em virtude da magnitude epidemiológica da manifestação mórbida, como por exemplo, hipertensão e diabetes.

Por meio da análise do planejamento realizado pelos estudantes, identificou-se que no nível terciário $10(17 \%)$ das atividades educativas compreenderam orientações específicas voltadas para os cuidados após alta hospitalar, com enfoque nos seguintes temas: cuidados pós-operatórios; utilização correta dos medicamentos; e, cuidados com a pele em pacientes com mobilidade física prejudicada.

Tabela 2 - Associação entre a temática geral da atividade de Educação em Saúde e o nível de atenção em saúde onde foi realizada. Fortaleza, CE, Brasil, 2015

\begin{tabular}{lcccc} 
Nível de atenção em saúde & \multicolumn{3}{c}{ Temática Geral } \\
\cline { 2 - 5 } & $\begin{array}{c}\text { Comportamentos } \\
\text { saudáveis }\end{array}$ & $\begin{array}{c}\text { Prevenção de } \\
\text { complicações } \\
\text { associadas a } \\
\text { doenças de base }\end{array}$ & $\begin{array}{c}\text { Orientação } \\
\text { de cuidados/ } \\
\text { tratamentos a } \\
\text { grupos específicos }\end{array}$ & $\begin{array}{c}\text { Total } \\
\mathbf{n}(\%)\end{array}$ \\
\hline Atenção Primária & $\mathbf{n}(\%)$ & $\mathbf{n}(\%)$ & $12(20)$ & $38(63)$ \\
\hline Atenção Secundária & $14(23)$ & $12(20)$ & $3(5)$ & $9(15)$ \\
\hline Atenção Terciária & - & $6(10)$ & $10(17)$ & $13(22)$ \\
\hline Total & - & $3(5)$ & $25(42)$ & $60(100)$
\end{tabular}

\section{DISCUSSÃO}

Educar na área da saúde é ir além da assistência curativa e preventiva, devendo reconhecer os usuários do serviço de saúde como seres humanos e depois como seres pensantes, que têm conhecimento de seus direitos e deveres e, desta forma, estimulá-los a lutarem por melhorias dos serviços de saúde e por mais qualidade de vida e dignidade ${ }^{(10)}$.

É recomendado que práticas de educação em saúde sejam estimuladas aos acadêmicos de enfermagem como forma de torná-los profissionais conscientes de sua importância sociopolítica, e assim mediadores na compreensão dos sujeitos como seres que têm o poder de modificar a realidade ${ }^{(11)}$.

A educação em saúde tem um papel importantíssimo diante do processo de ensino-aprendizagem, pois contribui para a autoformação do indivíduo, de modo que venha ensiná-lo a viver de maneira melhor, assumindo as suas responsabilidades em saúde e o seu papel de cidadão dentro de uma sociedade, além de contribuir para o aperfeiçoamento da função do educador ${ }^{(12)}$.

O enfermeiro e futuros enfermeiros precisam desenvolver habilidades como saber falar em público e coordenar um grupo destinado ao desenvolvimento da educação em saúde. O educador deve realizar suas atividades, quaisquer que sejam, de modo que o usuário entenda a mensagem a ser repassada, e, para isso, é fundamental uso de linguagem simples e acessível e conhecimento do público-alvo ${ }^{(13)}$.

Percebeu-se nesta pesquisa que poucas foram as atividades planejadas com enfoque para o estímulo de comportamentos saudáveis na população, independentemente da existência de doenças de base. A esse respeito, concorda-se que é preciso avançar para além do paradigma biologicista que focaliza a doença e suas causas, e colocar em prática a formatação do novo paradigma que visa, sobretudo, à manutenção do ser humano saudável, com enfoque para os determinantes sociais de saúde(2).

Foi constatado que o teatro é uma técnica fortemente lúdica que se constitui em um instrumento de empoderamento para a promoção da saúde, pois utiliza uma linguagem capaz de aprimorar as relações humanas e a comunicação, e extrapola as bases tradicionais das técnicas de educação em saúde, suscitando cenas cotidianas e trazendo a plateia para um papel mais ativo no processo educacional ${ }^{(14)}$. 
De fato, é preciso inovar quanto às técnicas utilizadas para a realização das práticas de educação em saúde, de modo que seja preterido o modelo tradicional, em que há uma transferência verticalizada do conhecimento e da informação, e passe a ser incentivado o modelo dialógico, não hierarquizado, e que haja valorização dos sujeitos na construção de comportamentos assertivos para melhoria da saúde ${ }^{(2)}$.

Sabe-se que há ainda fortes resquícios do modelo biomédico imbricado no planejamento e execução de atividades educativas em saúde ${ }^{(15)}$, representado principalmente pela maior ocorrência de práticas cuja temática geral gira em torno da prevenção de complicações associadas a doenças de base e orientações/tratamento de grupos específicos.

Por meio de uma pesquisa realizada em Portugal em cursos de graduação na área de saúde, constatou-se que, de modo geral, à medida que evoluem os períodos acadêmicos e a complexidade do conhecimento, os estudantes de enfermagem apresentam maior assimilação aos valores da educação em saúde, que são: social, salutogênico, holístico, equidade, autonomia e democrático(16).

Quanto ao cenário de realização das atividades, houve maior frequência na atenção primária, o que reforça a ideia de que as práticas de educação em saúde neste nível de atenção contribuem para a melhoria no autocuidado dos usuários do sistema de saúde, e fazem com que a população adquira conhecimentos e modifiquem sua opinião em relação à prevenção de doenças, reajustando consequentemente seus hábitos de vida, além de desenvolver nos usuários a autonomia diante do seu processo de cuidado em saúde ${ }^{(17)}$.

É importante destacar que independentemente do cenário onde a prática educativa aconteça, ela gera nos indivíduos que a praticam maior responsabilização pelo cuidar e fortalecem também sua autonomia, além da compreensão de que além de saber técnico-científico, a educação em saúde é um comprometimento social que visa o bem estar da população ${ }^{(18)}$.

Educar requer o entendimento que nós não somos apenas objetos da história, mas sujeitos da mesma $^{(19)}$. Estamos conscientes que não podemos eliminar os problemas de saúde, mas certamente podemos diminuir os danos que eles causam através da educação. O Brasil é um país que trata seus cidadãos de maneira predominantemente curativa, e é função dos docentes conscientizar acerca da importância do resgate de práticas de ensino que sejam centradas em ações preventivas não só na atenção primária, mas na atenção terciária, lembrando, sempre, que as práticas educativas em saúde são fundamentais em todos os níveis de atenção.

\section{CONCLUSÃo}

A análise das práticas educativas permitiu identificar que há o predomínio do modelo eminentemente preventivo nas propostas apresentadas pelos estudantes, e que a ludicidade evidenciada por meio de técnicas interativas e participativas, como o teatro, foram utilizadas com o objetivo de aproximar a clientela das atividades propostas.

Ao realizar a identificação das características das práticas educativas em saúde realizadas por estudantes de enfermagem, é possível inferir que o estudo contribui sobremaneira para repensar estratégias inovadoras do ensino sobre o tema, bem como demonstra a necessidade de maiores discussões do modelo de promoção da saúde como elemento central do ensino superior no Brasil.

Não acompanhar a execução das atividades educativas para avaliar o desempenho dos estudantes foi uma limitação identificada no estudo. Assim, sugere-se que pesquisas futuras possam avaliar longitudinalmente a prática da atividade educativa desde a sua concepção até os impactos gerados com a sua implantação junto à clientela.

\section{REFERÊNCIAS}

1. Silva MG, Fernandes JD, Teixeira, GAS, Silva RMO. Processo de formação do(a) enfermeiro(a) na contemporaneidade: Desafios e perspectivas. Texto Contexto Enferm.[Internet] 2010; 19(1) [acesso em 04 jun 
2015]. Disponível: http://dx.doi.org/10.1590/S0104-07072010000100021.

2. Salci MA, Maceno P, Rozza SG, da Silva DMGV, Boehs AE, Heidmann ITSB. Educação em saúde e suas perspectivas teóricas: algumas reflexões. TextoContexto Enferm. [Internet] 2013; 22(1) [acesso em 04 jun 2015]. Disponível: http://dx.doi.org/10.1590/S0104-07072013000100027.

3. Ferreira VF, Lopes MMB. Educação em saúde: desafios para uma prática inovadora. Rev Enferm UFPE on line. [Internet] 2013; 7 (n.esp) [acesso 18 jun 2015]. Disponível: http://www.revista.ufpe.br/revistaenfermagem/index. php/revista/article/download/4687/7328.

4. Herr GE, Kolankiewicz ACB, Berlezi EM, Gomes JS, Magnago TSBS, Rosanelli CP, et al. Avaliação de conhecimentos acerca da doença oncológica e práticas de cuidado com a saúde. Rev. Bras.Cancerol. 2013; 59(1): $33-41$.

5. Siqueira MS. Programa de educação em saúde para estudantes da escola estadual Tuiuti [monografia]. Porto Alegre (RS): Fundação Oswaldo Cruz; 2012.

6. Peduzzi M. Trabalho e educação na saúde: ampliação da abordagem de recursos humanos. Ciênc. Saúde Coletiva. [Internet] 2013; 18(6) [acesso em 15 jul 2015]. Disponível: http://dx.doi.org/10.1590/S1413-81232013000600005.

7. Girão RV, Braga ALS, Crhistóvam BP, Escudeiro CL, Lima VMF, Lopes CM. Educação em saúde sobre a dengue: contribuições para o desenvolvimento de competências. J. res.: fundam. care. online. [Internet] 2014; 6(1) [acessoem 18 jul 2015]. Disponível: http://dx.doi.org/10.9789/2175-5361.2014v6n1p38.

8. Jacobi CS, da Silva RM, Magnago TSBS, Prochnow A, Noal HC, Beuter M. Contribuições de ações extensionistas de educação em saúde no pós-operatório de cirurgias traumatológicas. R. Enferm. Cent. O. Min. 2013; 3(1): 60511.

9. Ministério da Saúde (BR). Conselho Nacional de Saúde. Diretrizes e normas regulamentadoras de pesquisa envolvendo seres humanos. Resolução n. 466, de 12 de dezembro de 2012. Brasília; 2012.

10. Oliveira RL, Santos MEA. Educação em Saúde da família: conhecimentos e práticas do enfermeiro.Revista Enfermagem Integrada. 2011;4(2):833-44.

11. Ferreira VF, da Rocha GOR, Lopes MMB, dos Santos MS, de Miranda SA. Educação em saúde e cidadania: revisão integrativa. Trab. educ. saúde. [Internet] 2014; 12(2) [acesso em 18 jul 2015]. Disponível: http://dx.doi. org/10.1590/S1981-77462014000200009.

12. Figueiredo MFS, Rodrigues-Neto JF, Leite MTS. Modelos aplicados às atividades de educação em saúde: revisão. Rev.bras. enferm. [Internet] 2010; 63(1) [acesso em 23 jul 2015]. Disponível: http://dx.doi.org/10.1590/ S0034-71672010000100019.

13. Fernandes MCP, Backes VMS. Educação em saúde: perspectivas de uma equipe da Estratégia Saúde da Família sob a óptica de Paulo Freire. Rev. bras. enferm. [Internet] 2010; 63(4) [acesso em 23 set 2015]. Disponível: http:// dx.doi.org/10.1590/S0034-71672010000400011.

14. Campos CNA, dos Santos LC, de Moura MR, de Aquino JM, Monteiro EMLM. Reinventando práticas de enfermagem na educação em saúde: teatro com idosos. Esc. Anna Nery. [Internet] 2012; 16(3) [acesso em 23 set 2015]. Disponível: http://dx.doi.org/10.1590/S1414-81452012000300023.

15. Pereira FGF, Caetano JÁ, Moreira JF, Ataíde MBC. Práticas educativas em saúde na formação de acadêmicos de enfermagem. CogitareEnferm. [Internet] 2015; 20(2) [acesso em 15 out 2015]. Disponível: http://dx.doi. org/10.5380/ce.v20i2.39767.

16. Carvalho AAS, de Carvalho GS, Rodrigues VMCP. Valores na Educação em Saúde e a formação profissional. Trab. educ. saúde. [Internet] 2012; 10(3) [acesso em 20 out 2015]. Disponível: http://dx.doi.org/10.1590/S198177462012000300010 .

17. Dantas MBP. Educação em saúde na Atenção básica: Sujeito, diálogo, intersubjetividade [tese]. Recife (PE): Fundação Oswaldo Cruz; 2010.

18. de Oliveira MR, Leonel ARA, Montezeli JH, Gastaldi AB, Martins EAP, Caveião C. Concepção de graduandos de enfermagem sobre a prática educativa em saúde em primeiros socorros. Rev Rene. 2015; 16(2) [acesso em 20 
out 2015]. Disponível: http://dx.doi.org/10.15253/2175-6783.2015000200003.

19. Freire P. Pedagogia da autonomia: saberes necessários à prática educativa. São Paulo: Paz e Terra; 1994. 